\title{
A definition of timetable stability for a long-term timetable
}

\author{
T. Graffagnino \& H. Labermeier \\ Swiss Federal Railways, Infrastructure, \\ Timetable and Offer, Switzerland
}

\begin{abstract}
Switzerland aims to invest 7 to 12 billion Swiss francs for the horizon 2030. These investments must bring with them an increase in the stability of the railway system. In order to evaluate the stability of different combinations of timetables and infrastructure projects, a stability assessment method has been developed by the Swiss Federal Railways. Based on the experience of computing the annual punctuality forecast, the evaluation of stability is made by computation of a punctuality forecast for each combination of future timetable and infrastructure project. Each forecast can then be compared using a set of quality goals, which are defined for 53 nodes and for the network as a whole. The timetable is then defined stable when all quality goals are achieved. With this method, the timetable stability is measurable which can ensure that the investment is made at the right place. This paper discusses the different models to assess timetable stability that are commonly found and describes the method used to make decisions on stability investments. To conclude, the results of the calibration, which is the first step applied to a real case, will be shown.

Keywords: timetable stability, stochastic simulation, Switzerland, cyclic timetable, punctuality.
\end{abstract}

\section{Introduction}

By the year 2030 an investment of between 7 and 12 billion Swiss francs is planned for the railway system. The Swiss railway authority defined four guiding principles, where the first is the following (original version translated from German into English) [1]: 
The development of the railway has to be carried out in relation to the demand during peak hour. Furthermore, the capacity of the network has to be increased to ensure the stability, the development and the maintenance of the network.

The Swiss Federal Railway (hereafter SBB) proposed the stability evaluation method that will be used to assess the different possible investments.

The main practical question was: how much stability is needed?

Hence the question, how to measure the stability in order to be able to decide how much is enough...

This paper describes how we answered this question, presents the stability evaluation method and the tools involved.

\section{Goal of the stability definition}

The goal is to compare, in terms of stability aspects, various combinations of offers (timetable options) and investments projects, under consideration of future maintenance conditions. The method is also able to evaluate the impact of longer dwelling time due to more passengers or new strategy for waiting times of connections. Based on these comparisons, decisions on investments for stability improvement will be made.

In that context, the definition of stability must support the investment decisions of millions of Swiss francs for the railway network.

\section{Timetable stability models}

Timetable stability is a very interesting research topic and numerous studies have been carried out on it (for example [2-6] or [7]). Usually, one has to build a model in order to simulate the interactions between the topology and the trains, and within the trains themselves. For that, different types of models have been developed (see also 3.2). Afterwards, the quality of the model has to be assessed. Finally, some examples of possible results are shown.

The focus of this paper is to propose a stability definition which can answer the following practical questions: where does the network need more stability, which investment brings how much more stability and how much stability will be enough for the network?

These questions are very important for the SBB indeed, and weren't so precisely answered until the development of the method presented here.

\subsection{A simplified view on stability}

In general, a timetable consists of a collection of train paths. Each train path is composed of a train composition, a collection of itineraries and commercial or service stops. The stability of a train is understood in practice as the probability of the train to remain on its planned train path. Inspired by [8], the stability can be represented with a small ball standing on a line: 


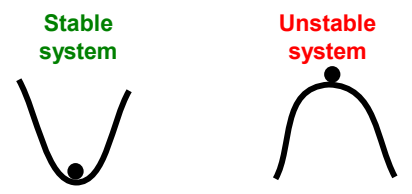

Figure 1: Simplified representation of stability.

A stable system will be capable of absorbing big perturbation (delays) and an unstable system will already be disturbed with small perturbation (delays). This already brings the next very important question for the assessment of the stability: how much perturbation must my system be able to absorb?

\subsection{Levels of detail of railway traffic models}

Railway traffic models can be categorised into three levels of detail: macro level, which is a high level and not a very precise model, where station are nodes and lines are arcs, micro level, which is a very detailed model including signals and interlocking logic, and finally, something in between, a so called "meso" level model, which is based on pre-computed detailed information on interlocking systems provided by a micro model and combined on a higher level model more similar to a macro level.

For the evaluation of timetable stability at SBB we are currently using two models: a micro level simulation model [9] to answer, besides other questions, regional stability questions and a meso level model to answer network-wide stability questions [10] based on stochastical computation.

\subsection{Type of traffic simulation}

Once a level of detail is set, then we can use it to simulate the railway traffic. Here, we can make a distinction between deterministic and stochastic models. On a deterministic model, like [9], at each time step, all train positions will be calculated and the position of each train is unique. In order to assess the system response on different delay scenarios, multiple simulations are needed. For example, Monte Carlo methods can be used to create different delay scenarios. With a stochastic model like [10], a given train has no unique position anymore, but has many positions at the same time, each position with a given probability of occurrence. Such a model is more like those used in particle physics research, where a particle has a different position at the same time...

\subsection{Stability evaluation based on deterministic models}

A stability evaluation with a deterministic model will evaluate how each train is able to absorb delays. For such methods, one has to define which primary delay, in the form of a given number of seconds, has to be considered on each train of the simulation. The model will then deduce secondary delays for that particular case. The quality of the results is highly dependent on the choice of primary delay scenarios. These simulations are very time consuming and only realistic scenario 
delays have to be assessed. At SBB we develop our delay scenarios based on real traffic data which are visualised and exported with our Software Open Timetable [12]. Because we use real traffic data, we reduce the risk of evaluating non-realistic delay scenarios.

For different delay scenarios, the results of the simulation can be represented with the following figure:
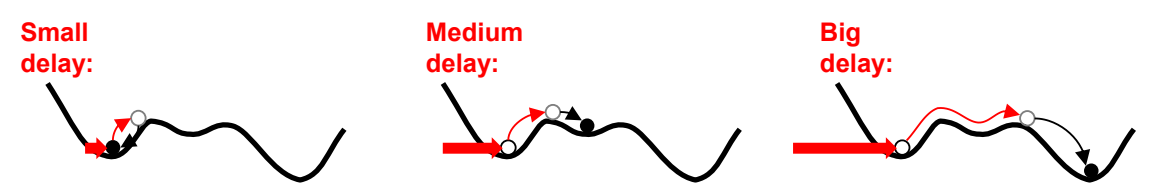

Figure 2: Results with deterministic models.

Decisions based on such results mean to fix a delay scenario that has to be absorbed and to fix a threshold for what you accept and what you don't accept. The threshold can be represented with the simplified following figure:

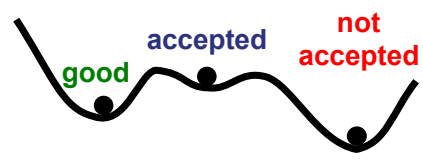

Figure 3: Threshold for acceptance.

In a simulation, for a given delay scenario, if the ball ends in a non-accepted state, then you will declare it as unstable and it is not accepted.

At SBB this type of simulation is mainly used to support experts in order to evaluate the sensibility of different delay situations for a given region.

\subsection{Stability evaluation based on stochastic models}

A stability evaluation with a stochastic model will evaluate all train positions of each train at once. For such methods, one has to define which primary delay, in the form of a delay probability function, has to be considered on each train. The model will then compute the secondary delay in the form of a delay probability function for each train on the network. Which primary delay to consider will be discussed in section 7 of this paper.

For a given primary delay scenario, one has to decide what to accept and not to accept. This is a bit more abstract for stochastic results. At the end of a simulation, for a given ball, the final position is not unique, but the ball can be at all positions with a given probability. This can be represented with the following simplified figure (figure 4).

In this figure we represented two different results, one on the right with a $10 \%$ probability for the far right position and another with a $20 \%$ probability for the far 
right position. For this example, we assumed that the far right position must have a probability of occurrence not higher than $15 \%$. Hence, on the right hand side of Figure 4 , the probability of $20 \%$ is too high and the goal is not achieved.
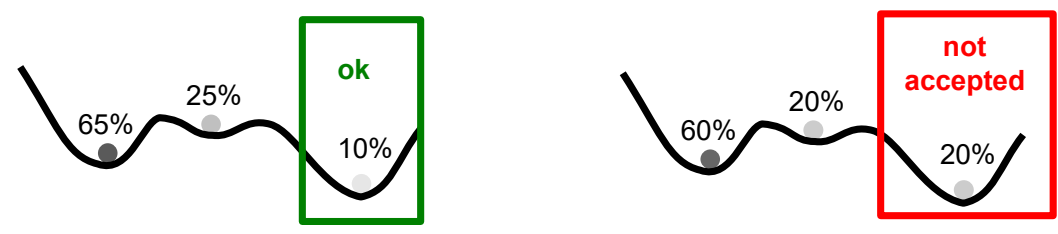

Figure 4: Results with stochastic models.

\section{Definition of timetable stability}

The stability evaluation of a train run with a stochastic model was described in point 3.5. If one has to consider more than one train, the question of the sum of the decision has to be solved also: if we consider that we don't have two different cases of the same train on Figure 4 but two separate train runs, what decision can be made? Is it then stable or not, when one train achieves the goal and the other doesn't?

Because we have to evaluate the long-term timetable for 2030, SBB has to consider a collection of more than ten thousands train paths! In such a case, the decision regarding what to accept and what not to accept has to be made for all train paths at the same time.

To answer this question, we did simulate the railway traffic on the whole network with the model OnTime, a stochastic meso model [10]. We decided to fix the threshold at 3 minutes which is the one used for punctuality measures and grouped the different train paths, de facto producing sums of decision, in the same manner as we do for the yearly punctuality figures. Having done that, the decision of what is acceptable and what is not acceptable for the year 2030 can be naturally answered. Having to define a given percentage of trains which have to remain under 3 minutes, we decided to fix the acceptance at the punctuality goal of 2015 (which wasn't achieved that year): $92 \%$ of the arrivals have to have a delay of less than 3 minutes. This is measured on 53 key nodes. In order to ensure the stability of every node, we then fixed a minimal punctuality value to achieve. And for every node, we also fixed a minimal punctuality value to achieve for regional and longdistance traffic. Having fixed different minimal punctuality values for different collections of train runs or sums of decision, we can ensure the stability of the timetable, which leads us to the following definition: The timetable is stable when all punctuality goals are achieved.

With such a web of stability measurement points, we can then be sure that the effects of each investment will be identified and evaluated. Furthermore, having built a bridge to the train punctuality measure, we can naturally identify when there are enough investments for stability thus avoiding over-investments. 


\section{Practical results}

In Switzerland, due to the fact that we have a cyclic timetable ("Taktfahrplan"), a conflict-free timetable structure between 06:00 and 21:00 already known as a long-term timetable. It is our way to ensure that future investments will be made at the right place in order to make the new demanded train paths possible. We engaged stochastic meso Model Tool OnTime [10] in order to simulate future railway traffic on the Swiss network. We went through various steps and challenges which are described here.

\subsection{Big picture of the process}

In order to evaluate and assess the stability of the future timetables we went through various steps which can be mapped in figure 5 .

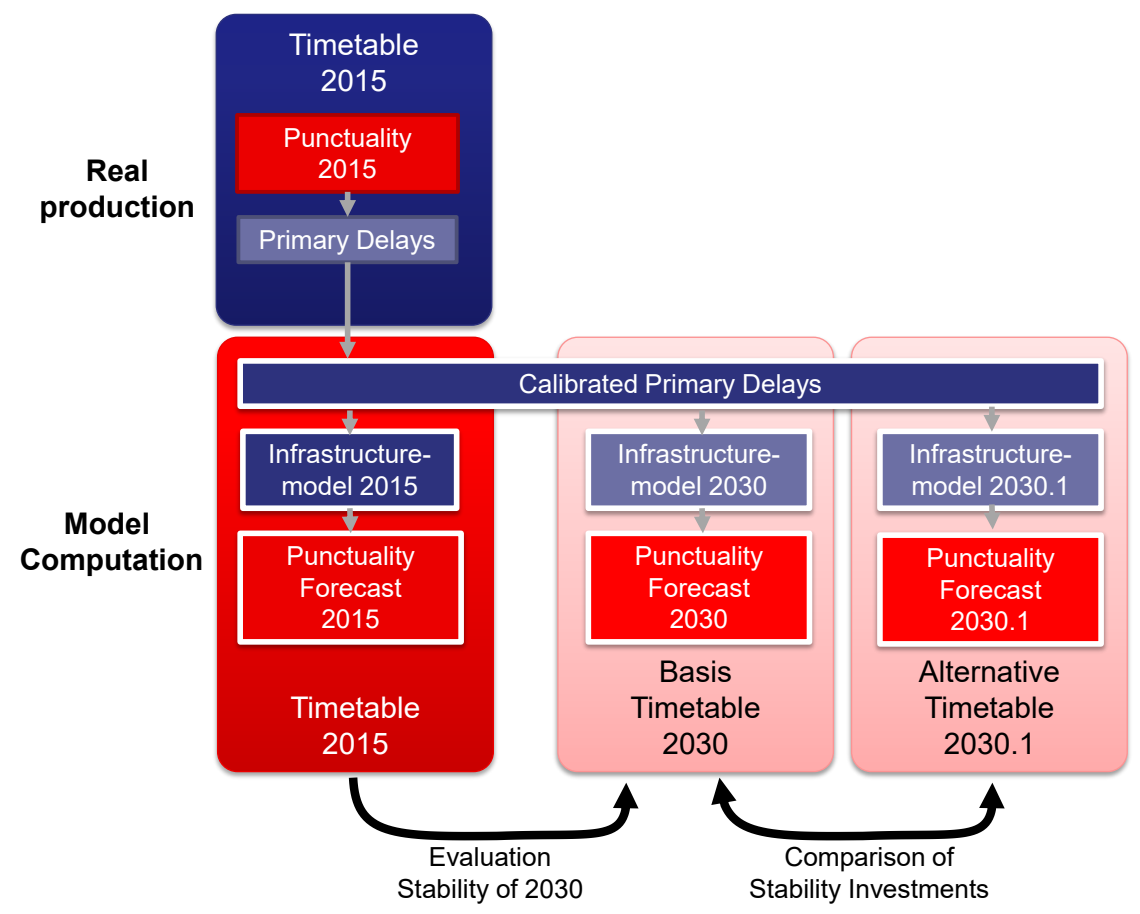

Figure 5: Evaluation process.

\subsection{Preparation of the infrastructure model}

To begin, the meso model was built up by defining all parameters needed such as all headways, separation times for conflicting itineraries, connections and connections parameters between trains, splitting/joining/turning of rolling stocks including necessary minimal times. The quality of these parameters is crucial to 
compute useful results, otherwise one might minimise or maximise stability problems on the network.

\subsection{Real production and reference year}

Then we had to choose a year of reference where real production data are available and the punctuality is known. In this case we agreed upon the timetable of year 2015. We then extracted the primary delays from the past traffic data of 2015 and produced the first lists of primary delays. More details on the extraction of primary delays is to be found in the following paper from Labermeier [13]. Then we computed the real measured punctuality on the network for all the key nodes, with the distinction between peak hour and off-peak hour, separating regional trains from long-distance trains.

Furthermore, the timetable quality is also important for the evaluation. Because the time margins are crucial for the stability assessment, the calculation of the technical time for the timetable have to be of good quality.

\subsection{Calibrated primary delays}

The calibration consists of tuning the lists of primary delays in order for the model to compute a punctuality forecast with a minimal deviation to the measured punctuality. After the first extraction of primary delay as explained in [13], the first forecast is made. For the nodes where the forecast is too bad compared to the measured punctuality, the calibration consists of reducing the primary delay of the trains heading to this node. For the nodes where the forecast is too good, the primary delays of the related trains will be increased. The calibration was considered as achieved, if within a group (node, train type, period of the day) with at least 30 arrivals, the deviation between the measures of punctuality and its forecast was not bigger than 3\%-point for the punctuality of the arrivals at $3 \mathrm{~min}$.

\section{Calibration results}

We were able for almost all groups to achieve our declared quality goal (to be found under 5.4). One example of practical challenge was the correct calibration of the peak hours for large nodes. During the morning peak hour, the delays are on trains running upwards on a large node. For yearly forecast in Switzerland, the train directions can be recognised by the parity of the train numbers. For the longterm timetable, the train numbers are not numeric but alphanumeric which makes it impossible for them to be used in the model to distinguish between the train running direction. Due to that, it is impossible to achieve the same calibration quality for the long-term timetable as for the yearly timetable. The calibration figures for all groups with more than 30 arrivals are summarised in table 1 .

For the entire network, we also produced graphics for the line in order to check the quality of the model with our train traffic visualisation tool Open Timetable [11] (see figure 6). 
Table 1: Calibration results.

\begin{tabular}{|c|c|c|c|c|}
\hline & $\begin{array}{l}\text { Morning } \\
\text { peak hour }\end{array}$ & Day & $\begin{array}{c}\text { Evening } \\
\text { peak hour }\end{array}$ & Total \\
\hline \multicolumn{5}{|l|}{ Regions } \\
\hline RWT & $0.09 \%$ & $0.76 \%$ & $-0.41 \%$ & $0.46 \%$ \\
\hline RME & $0.99 \%$ & $-0.09 \%$ & $0.84 \%$ & $0.31 \%$ \\
\hline RSD & $-0.89 \%$ & $1.52 \%$ & $1.07 \%$ & $0.94 \%$ \\
\hline ROT-ZUE & $0.33 \%$ & $0.18 \%$ & $1.10 \%$ & $0.43 \%$ \\
\hline ROT-SG & $0.07 \%$ & $-0.09 \%$ & $0.73 \%$ & $0.11 \%$ \\
\hline Schweiz & $0.43 \%$ & $0.24 \%$ & $0.69 \%$ & $0.40 \%$ \\
\hline \multicolumn{5}{|c|}{ Short list Key-Nodes } \\
\hline Biel & $-2.86 \%$ & $-0.88 \%$ & $-1.45 \%$ & $-1.31 \%$ \\
\hline Genève & $0.65 \%$ & $1.19 \%$ & $-1.25 \%$ & $0.66 \%$ \\
\hline Lausanne & $-3.18 \%$ & $-0.80 \%$ & $-2.66 \%$ & $-1.51 \%$ \\
\hline Visp & & $2.30 \%$ & $-1.29 \%$ & $1.06 \%$ \\
\hline Basel SBB & $1.34 \%$ & $1.09 \%$ & $-0.53 \%$ & $0.90 \%$ \\
\hline Bern & $0.92 \%$ & $-1.08 \%$ & $-1.60 \%$ & $-0.82 \%$ \\
\hline Luzern & $-2.72 \%$ & $-0.91 \%$ & $-1.91 \%$ & $-1.49 \%$ \\
\hline Olten & $-0.15 \%$ & $0.19 \%$ & $-0.18 \%$ & $0.04 \%$ \\
\hline Bellinzona & $-1.25 \%$ & $2.05 \%$ & $2.38 \%$ & $1.56 \%$ \\
\hline Winterthur & $0.47 \%$ & $1.05 \%$ & $-0.27 \%$ & $0.65 \%$ \\
\hline Zürich HB & $2.00 \%$ & $-0.90 \%$ & $0.73 \%$ & $0.00 \%$ \\
\hline Sargans & & $-0.40 \%$ & $-0.46 \%$ & $-0.32 \%$ \\
\hline St. Gallen & $0.41 \%$ & $-1.33 \%$ & $-1.79 \%$ & $-1.11 \%$ \\
\hline
\end{tabular}

These are the results of the forecast obtained after a simulation. In this graphic, we can see long-distance, regional and freight trains between Olten und Zürich for one hour between 06:00 and 07:00. It is very challenging to ensure the quality of such a network-wide model. Being able to produce a line graphic for all major lines of the Swiss network with real train traffic data and forecasted train traffic data is the key to success. During the calibration process, we can identify where possible deviations between the forecasted and the real traffic are, in order to iteratively adapt the primary delay for reducing these deviations.

\section{Evaluation of stability for Timetable 2030}

The correct amount of primary delay to expect is quite difficult to say 15 years in advance. But because we have to show that the new timetable will be better, it is enough to prove that under the same conditions, the new timetable is better. Hence allowing us to use the same amount of primary delays as calibrated with the reference year 2015. If the punctuality figures of 2030 compared to 2015 are better, then a better stability is to be expected for the new timetable thanks to the considered investments. Another point will be to compare different investment projects for 2030. For that, under the same primary delays, two forecast for 2030 will be compared. 


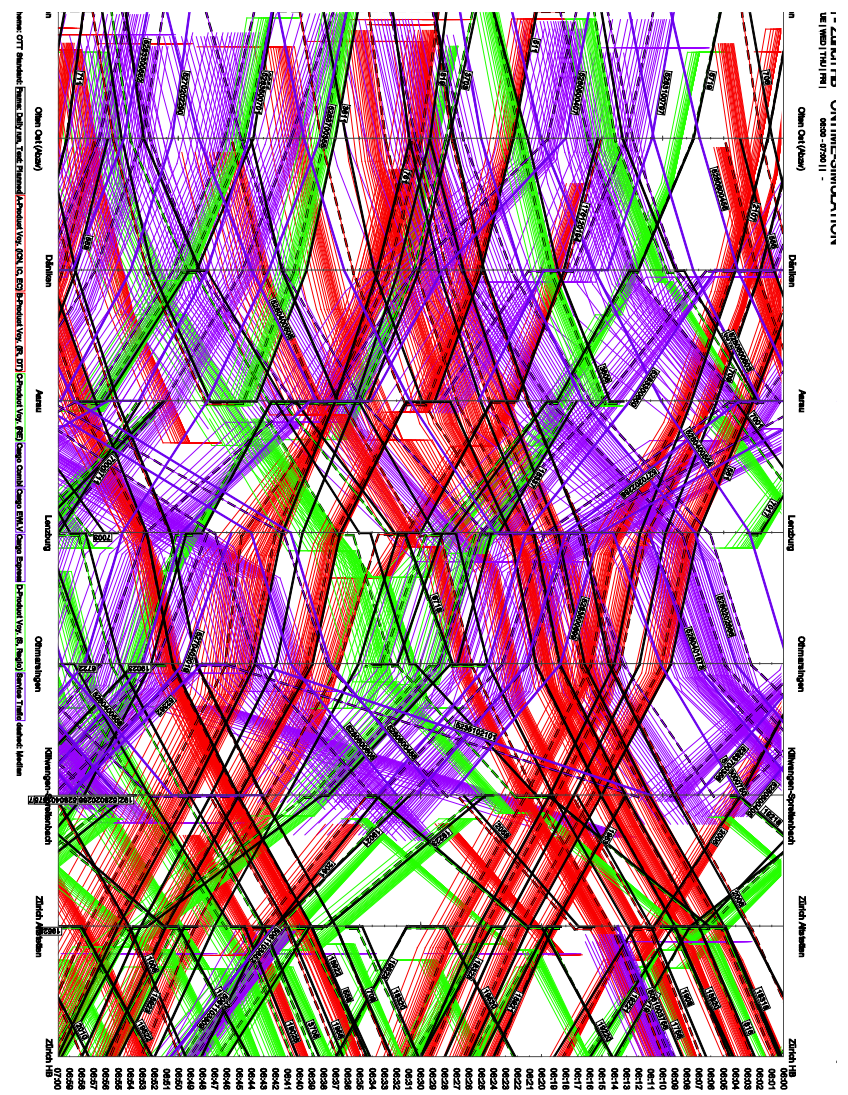

Figure 6: Forecast results as line.

\section{Conclusions and further steps}

The practical evaluation of the stability of long-term timetables is a very challenging topic. Thanks to this method and the quality of the available detailed timetable information for the long term in Switzerland, we are able to precisely evaluate and compare the impact of each foreseen investment or timetable changes. Having been able to fix a clear goal for the stability investment in the form of a well-known train punctuality figure was a very big step forward. Having successfully closed the calibration phase, we will now focus our work on computing the forecasts for future timetables and comparing them. 


\section{References}

[1] Swiss Federal Office of Transport, Dokumentation Planungsgrundlagen STEP Ausbauschritt 2030 - Bericht, Bern, 2014, http://www.bav.admin.ch /fabi/04578

[2] Thorsten B., Ausgewählte Aspekte der Verspätungsfortpflanzung in Netzen, PhD, RWTH, Aachen, Germany, 2011

[3] Goverde R., Punctuality of Railway Operations and Timetable Stability Analysis, PhD, TU Delft, Netherlands, 2005

[4] Noordeen M., Stability Analysis of Cyclic Timetables for Highly Interconnected Rail Network, PhD, EPFL, Lausanne, Switzerland, 1995

[5] Hürlimann D., Objektorientierte Modellierung von Infrastrukturelementen und Betriebsvorgängen Im Eisenbahn, PhD, ETHZ, Zürich, Switzerland, 2002

[6] Herrmann T., Stability of Timetables and Train Routings through Station Regions, PhD, ETHZ, Zürich, Switzerland, 2006

[7] Burkolter D.M., Capacity of Railways in Station Areas using Petri Nets, ETHZ, Zürich, Switzerland, 2005

[8] Definition of Stability in German, Wikipedia ${ }^{\circledR},(2013$, August 22), Online on the 30.09.2015, https://de.wikipedia.org/wiki/Stabilit\%C3\%A4t

[9] OpenTrack, http://www.opentrack.ch/

[10] OnTime, http://www.trafit.ch/en/ontime

[11] Open Timetable, http://www.via-con.de/ott

[12] Graffagnino, T., "Ensuring timetable stability with train traffic data", In: Brebbia, C.A., Tomii, N., Mera, J.M., Ning, B., Tzieropoulos, P. (eds.), Computers in Railways XIII, pp. 427-438, WIT Press, Southampton, 2012

[13] Labermeier H., On the Dynamic of Primary and Secondary Delay, Proceedings of IAROR, Copenhagen, 2013 